\title{
About the Series - Sur la collection - Zur Buchreihe
}

The avant-garde and modernism take centre-stage within European academia today. The experimental literatures and arts in Europe between ca. 1850 and 1950, and their aftermath, figure prominently on curricula, while modernism and avant-garde studies have come to form distinct yet interlocking disciplines within the humanities in recent years. These disciplines take on various guises on the continent. Within French and German academia, "modernism" remains a term rather alien - "die Moderne" and "modernité" coming perhaps the closest to what is meant by "modernism" within the English context. Here, indeed, modernism has acquired a firm place in research, signaling above all a period in modern poetics and aesthetics, roughly between 1850 and 1950, during which a revolt against prevalent traditions in art, literature and culture took shape. Similarly, the term "avant-garde" comes with an array of often conflicting connotations. For some, the avant-garde marks the most radically experimental arts and literatures in modernism from the nineteenth century onward - the early twentieth-century vanguard movements of Futurism, Expressionism, Dada and Surrealism, among others, coinciding with the avant-garde's most "heroic" phase. For others, the avant-garde belongs to a cultural or conceptual order differing altogether from that of modernism - the vanguard exploits from the 1950s onward marking that avant-garde arts and literatures can also perfectly abide outside modernism.

European Avant-Garde and Modernism Studies, far from aiming to reduce the complexity of various European research traditions, aspires to embrace the wide linguistic, terminological and methodological variety within both fields. Publishing an anthology of essays in English, French and German every two years, the series aims to compare and relate French, German and British, but also Northern and Southern as well as Central and Eastern European findings in avant-garde and modernism studies.

Collecting essays stemming in large part from the biennial conferences of the European Network for Avant-Garde and Modernism Studies (EAM), books in this series do not claim to exhaustiveness. Rather, they aim to raise questions, to provide partial answers, to fill lacunae in the research, and to stir debate about the European avant-garde and modernism throughout the nineteenth and twentieth centuries and into the twenty-first. The series attaches great value to interdisciplinary and intermedial research on experimental aesthetics and poetics, and intends to encourage an interest in the cultural dimensions and contexts of the avant-garde and modernism in Europe. 
L'avant-garde et le modernisme occupent actuellement une place majeure dans les universités européennes. Les arts et les littératures expérimentaux en Europe de 1850 à 1950 et au-delà font partie intégrante des programmes universitaires, tandis que les recherches sur l'avant-garde et le modernisme sont devenues, à l'intérieur des sciences humaines, des disciplines à part entière mais solidaires l'une de l'autre. Ces disciplines varient néanmoins à travers le continent. Dans les universités françaises et allemandes, la notion de " modernisme » reste plutôt étrangère : les notions de " modernité » et de « die Moderne » s'utilisent sans doute davantage pour ce que désigne la notion de " modernism » dans le contexte anglophone. Dans la recherche anglophone, en effet, la notion de « modernism » a acquis une certaine stabilité : elle désigne avant tout une période de la modernité poétique et esthétique, approximativement entre 1850 et 1950, au cours de laquelle a pris forme une révolte contre les traditions artistiques, littéraires et culturelles prédominantes. De la même façon, la notion d' ' avant-garde " prend des connotations divergentes, souvent conflictuelles. Pour certains, l'" avant-garde » désigne les arts et les littératures les plus radicalement expérimentaux qui se développent à l'intérieur du modernisme à partir du $19^{\text {éme }}$ siècle. Dans ce cas, les mouvements avant-gardistes du début du $20^{\text {ème }}$ siècle - dont le futurisme, l'expressionisme, le dadaïsme et le surréalisme - correspondent à la phase avant-gardiste la plus « héroïque ». Pour d'autres, l'avant-garde appartient à un ordre culturel et conceptuel entièrement différent du modernisme. Dans cette perspective, l'avant-garde survit au modernisme, comme en témoigne la permanence d'une sensibilité avant-gardiste après 1950.

Loin de vouloir réduire la complexité et la variété des traditions de recherche européennes, la collection Études sur l'avant-garde et le modernisme en Europe vise à embrasser la grande diversité linguistique, terminologique et méthodologique à l'intérieur de ces deux domaines de recherche. Par la publication d'un volume d'essais en anglais, en français et en allemand tous les deux ans, la collection souhaite comparer et mettre en rapport les résultats issus des traditions de recherche française, anglaise et allemande, mais également d'Europe nordique et méridionale, centrale et orientale. La collection rassemble les travaux les plus novateurs et les plus stimulants de la recherche actuelle et se consacre à l'étude de l'avant-garde et du modernisme européens au cours des dix-neuvième et vingtième siècles.

Le premier objectif de cette collection est de rassembler une sélection des textes présentés lors des rencontres bisannuelles due Réseau européen de recherche sur l'avant-garde et le modernisme (EAM). En ce sens, son ambition est moins d'épuiser un sujet que de soulever les questions, de suggérer quelques réponses provisoires, de combler certaines lacunes dans la recherche et, plus 
généralement, de maintenir vivant le débat sur l'avant-garde et le modernisme européens au cours des $19^{\text {ème }}, 20^{\text {ème }}$ et $21^{\text {ème }}$ siècles. La collection attache beaucoup d'importance à la recherche interdisciplinaire et intermédiale sur les esthétiques et les poétiques expérimentales et se propose de stimuler l'intérêt pour les dimensions culturelles et contextuelles de l'avant-garde et du modernisme en Europe.

Forschungsinitiativen zum Thema Avantgarde und Moderne nehmen in der europäischen Forschungslandschaft weiterhin zu. Die experimentellen Literaturen und die Künste in Europa zwischen ca. 1850 und 1950 und ihre Nachwirkungen sind als Lehr- und Forschungsbereiche an den europäischen Forschungsinstitutionen und in den Lehrplänen heutzutage nicht mehr wegzudenken. Avantgarde und Moderne haben sich in den letzten Jahrzehnten zu unterschiedlichen, aber mehrfach miteinander verzahnten Forschungsgebieten entwickelt. Innerhalb der französischen und deutschen akademischen Welt bleibt der Sammelbegriff „modernism“ weniger geläufig - „die (klassische) Moderne“ und „modernité" fungieren hier als nahe liegende Äquivalente zu demjenigen, was im internationalen Kontext als eine zeitliche und räumliche Ko-Okkurenz künstlerischer Ausdrucksformen und ästhetischer Theorien namhaft gemacht werden kann, die ungefähr zwischen 1850 und 1950 angesiedelt werden kann. Auf ähnliche Weise entfaltet die Bezeichnung „Avantgarde“ eine Reihe häufig widersprüchlicher Konnotationen. Für manche bedeutet die Avantgarde den radikalsten experimentellen Bruch der Künste und Literaturen mit den Darstellungs- und Erzählkonventionen des 19. Jahrhundert: im frühen 20. Jahrhundert zeugen davon Avantgardebewegungen wie Futurismus, Expressionismus, Dada und Surrealismus, Strömungen, die als die „emphatische“ Phase der Avantgarde bezeichnet werden können. Für andere gehört die Avantgarde zu einem kulturellen Umfeld, das sich, durchaus im Bunde mit der Klassischen Moderne, der Erneuerung ästhetischer Konventionen verschreibt.

Die Buchreihe Studien zur europäischen Avantgarde und Moderne möchte der Komplexität der unterschiedlichen europäischen Forschungstraditionen gerecht werden und strebt danach, die breite linguistische, terminologische und methodologische Vielfalt abzudecken. Anhand einer zweijährlichen Sammlung von Beiträgen in englischer, französischer und deutscher Sprache möchte die Reihe nicht nur die französisch-, deutsch- und englischsprachigen, sondern auch die nord-, süd-, zentral- und osteuropäischen Ergebnisse der Avantgarde- und Moderne-Forschung einbeziehen.

Die Aufsatzsammlungen der Reihe, die größtenteils aus Beiträgen von den zweijährlichen Konferenzen des Europäischen Netzwerks für Studien zu Avantgarde und Moderne (EAM) bestehen, erheben keinen Anspruch auf Vollständig- 
keit. Ihr Ziel ist es vielmehr, Fragen zu stellen, einige Antworten vorzuschlagen, Forschungslücken zu schließen und Debatten über die europäische Avantgarde und die Moderne im neunzehnten, zwanzigsten und einundzwanzigsten Jahrhundert auszulösen. Die Studien zur europäischen Avantgarde und Moderne legen viel Wert auf die interdisziplinäre und intermediale Erforschung experimenteller Ästhetiken/Poetiken und setzen es sich zum Ziel, das Interesse an den kulturellen Zusammenhängen und Kontexten der Avantgarde und der Moderne in Europa anzuregen.

David Ayers and Sascha Bru

Canterbury \& Leuven 2020

Previous books in this series:

Europa! Europa? The Avant-Garde, Modernism, and the Fate of a Continent, ed. by Sascha Bru, Jan Baetens, Benedikt Hjartarson, Peter Nicholls, Tania Ørum and Hubert van den Berg (2009).

Regarding the Popular: Modernism, the Avant-Garde and High and Low Culture, ed. by Sascha Bru, Lawrence can Nuijs, Benedikt Hjartarson, Peter Nicholls, Tania Ørum and Hubert van den Berg (2011).

The Aesthetics of Matter: Modernism, the Avant-Garde and Material Exchange, ed. by Sarah Posman, Anne Reverseau, David Ayers, Sascha Bru and Benedikt Hjartarson (2013).

Utopia: The Avant-Garde, Modernism and (Im)possible Life, ed. by David Ayers, Benedikt Hjartarson, Tomi Huttunen and Harri Veivo (2015).

Beyond Given Knowledge: Investigation, Quest and Exploration in Modernism and the Avant-gardes, ed. by Harri Veivo, Jean-Pierre Montier, Françoise Nicol, David Ayers, Benedikt Hjartarson and Sascha Bru (2018). 